\title{
MRI Shows More Severe Hippocampal Atrophy and Shape Deformation in Hippocampal Sclerosis Than in Alzheimer's Disease
}

\author{
C. Zarow, ${ }^{1}$ L. Wang, ${ }^{2}$ H. C. Chui, ${ }^{3}$ M. W. Weiner, ${ }^{4}$ and J. G. Csernansky ${ }^{5}$ \\ ${ }^{1}$ Rancho Los Amigos National Rehabilitation Center, University of Southern California, 7601 E Imperial Hwy., Medical Science Bldg., \\ Room 26 Downey, CA 90242, USA \\ ${ }^{2}$ Departments of Psychiatry and Behavioral Sciences and Radiology, Northwestern University Feinberg School of Medicine, 710 N. Lake \\ Shore Drive, Abbott Hall 1312, Chicago, IL 60611, USA \\ ${ }^{3}$ Department of Neurology, University of Southern California, 1510 San Pablo Street, Suite 618, Los Angeles, CA 90033, USA \\ ${ }^{4}$ Center for Imaging of Neurodegenerative Disease, University of California, 4150 Clement Street, San Francisco, CA 94121, USA \\ ${ }^{5}$ Department of Psychiatry and Behavioral Sciences, Northwestern University Feinberg School of Medicine, Chicago, IL 60611, USA
}

Correspondence should be addressed to C. Zarow, zarow@usc.edu

Received 24 November 2010; Accepted 16 February 2011

Academic Editor: G. B. Frisoni

Copyright $\odot 2011$ C. Zarow et al. This is an open access article distributed under the Creative Commons Attribution License, which permits unrestricted use, distribution, and reproduction in any medium, provided the original work is properly cited.

While hippocampal atrophy is a key feature of both hippocampal sclerosis (HS) and Alzheimer's disease (AD), the pathology underlying this finding differs in these two conditions. In $\mathrm{AD}$, atrophy is due primarily to loss of neurons and neuronal volume as a result of neurofibrillary tangle formation. While the etiology of HS is unknown, neuron loss in the hippocampus is severe to complete. We compared hippocampal volume and deformations from premortem MRI in 43 neuropathologically diagnosed cases of HS, AD, and normal controls (NC) selected from a longitudinal study of subcortical ischemic vascular disease (IVD Program Project). HS cases $(n=11)$ showed loss of neurons throughout the rostral-caudal extent of the hippocampus in one or both hemispheres. AD cases $(n=24)$ met NIA-Reagan criteria for high likelihood of AD. Normal control cases $(n=8)$ were cognitively intact and showed no significant AD or hippocampal pathology. The mean hippocampal volumes were significantly lower in HS versus $\mathrm{AD}$ groups $(P<.001)$. Mean shape deformations in the CA1 and subiculum differed significantly between $\mathrm{HS}$ versus $\mathrm{AD}$, HS versus $\mathrm{NC}$, and $\mathrm{AD}$ versus NC $(P<.0001)$. Additional study is needed to determine whether these differences will be meaningful for clinical diagnosis of individual cases.

\section{Introduction}

Hippocampal sclerosis (HS) is a highly prevalent pathologic lesion, found in approximately $15 \%$ of elderly dementia cases in autopsy series [1]. It is a common cause of memory loss in late life, but is rarely diagnosed before autopsy. HS is characterized by selective neuronal loss with gliosis in the absence of cystic cavitation, involving the CA1 sector of the hippocampus and often extending into the subiculum. A sharp demarcation is often noted histologically between affected and adjacent normal hippocampal subfields. HS can affect one or both hemispheres and can be focal or widespread in its rostral-caudal extent [2]. HS is often accompanied by other types of pathology, but may occur as a relatively isolated finding [1]. The pathogenesis of HS is not well understood, and ischemia/hypoxia, neurodegeneration, or a combination of these nonmutually exclusive processes has been postulated.

A clinical diagnosis of HS is rarely made in late life. Most HS cases are discovered at autopsy and were diagnosed clinically as AD. The typical hallmarks of $\mathrm{HS}$ seen in early adulthood (namely, partial complex seizures and T-2 hyperintensity in the hippocampus) are absent in late-onset HS [3-5].

While hippocampal atrophy is the sine qua non of $\mathrm{AD}$, it is also observed in HS. Late-life HS is associated with hippocampal atrophy on MRI, both in vivo [3-5] and postmortem [6]. We previously reported MRI hippocampal 
volume to be independently associated with $\mathrm{HS}$ and AD pathology $[3,4]$. Thus, it has been difficult to distinguish HS from AD based on cross-sectional structural MRI.

Recently, automated methods for neuromorphometry have been developed, which allow for the precise statistical modeling of neuroanatomical surfaces [7]. Diffeomorphic mapping applied to the hippocampal surface has been successful in distinguishing $\mathrm{AD}$ from normal aging [8] and in predicting the conversion of cognitively normal subjects to very mild dementia [9]. In the present study, we used diffeomorphic mapping to compare patterns of deformation of the hippocampal surface in subjects with HS and $\mathrm{AD}$ compared to controls. Using nonbiased stereology counting methods, we previously noted greater neuron loss in $\mathrm{HS}$ than AD, especially in CA1 [10]. Therefore we used diffeomorphic mapping to test the hypothesis that HS could be distinguished from AD based on the severity of deformations of the hippocampal surface near the CA1 subfield.

\section{Materials and Methods}

2.1. Sample Selection. Autopsy cases were obtained from the Ischemic Vascular Dementia Program Project, a prospective, longitudinal study of subjects with subcortical ischemic vascular disease (IVD), Alzheimer's disease (AD), and cognitively normal elderly subjects (NC). The total available autopsy sample consists of 146 cases obtained over the 11 year span of 1997 to 2007. For this study, we considered 100 autopsy cases with bilateral hippocampi and MRI available for review.

Cases were evaluated for neurofibrillary tangle load (Braak \& Braak score), neuritic plaque burden (CERAD rating), Lewy bodies (McKeith Lewy body score), and vascular lesions including cystic, lacunar, and microinfarcts. HS was evaluated with the H\&E stain. At a minimum, two levels of the hippocampus were reviewed for each hemisphere: the level of the pes and at the level of the lateral geniculate nucleus. More commonly, the entire rostral-caudal extent of the hippocampus from pes to tail was evaluated. The severity of HS was scored as "none, focal, or complete," based on the extent the hippocampal involvement. HS was rated "none" when there was no HS, "focal" when HS was limited to a portion of a CA sector at a single level of the hippocampus, and "complete" when the HS involved the entire pyramidal layer of CA1 and/or subiculum through the rostral-caudal extent of the hippocampus.

Of 100 cases with bilateral hippocampi available for review, 31 had HS. HS cases selected for this study ( $n=$ 11) had complete hippocampal sclerosis in one or both hemispheres, few or no tangles (Braak and Braak score < III), or neuritic plaques (CERAD = none or sparse). Cases with focal HS $(n=3)$ or hippocampal infarcts $(n=4)$ were excluded as were HS cases with AD pathology $(n=13)$ due to numerous tangles in the hippocampus.

The AD cases $(n=24)$ included in this study had a Braak and Braak stage of $\mathrm{V}$ or VI and a CERAD plaque score of moderate or frequent. AD cases with HS or other co-morbidities were excluded.

NC cases $(n=8)$ were cognitively normal (clinical dementia rating scale, $\mathrm{CDR}=0$ ) at last clinical evaluation prior to death and had no significant pathology at autopsy, namely, Braak and Braak stage < III, CERAD neuritic plaque score of none or sparse, Lewy body score of 0 , and no vascular lesions. All cases meeting these criteria were included. Pathologically normal cases which were not cognitively normal $(\mathrm{CDR}>0)$ were excluded $(n=7)$.

2.2. Imaging. All imaging was performed on a 1.5 Tesla MR system (Siemens Vision System, Germany), using a standard head coil. Structural MRI included volumetric T1-weighted magnetization-prepared rapid acquisition gradient-echo (MPRAGE) image, a multislice proton density, and T2-weighted images based on a dual-echo sequence.

Hippocampal surfaces in each subject were obtained using template-based (UCSF template) high-dimensional brain-warping algorithm (Medtronic Surgical Navigation Technologies, Louisville, CO), which was created from MRI data acquired from five female and five male volunteers, 57 to 94 years of age, mean age 70.5 for all 11.1 years). Details of this method have been described elsewhere [11].

Hippocampal surface zones on the UCSF template surfaces corresponding to underlying subfields were transferred from an existing source (WUSM template) $[9,12]$ using surface matching techniques [13]. Subfields analyzed correspond to CA1, the subiculum, and a combined subfield comprising combined deformations for CA2, CA3, CA4, and the dentate granule cell layer. Hippocampal surface zones in each subject were obtained by the above brain warping maps. This procedure has been shown to have high intraclass correlation coefficients of the areas of the three surface zones (CA1 - 0.97; subiculum -0.97; combined -0.90), comparing manually outlined hippocampal surface zones with the surface zones mapped from the template [12]. Because the transformations from the template to each subject were one to one and onto, all subjects were inregistration with respect to the template. Thus, the different zones on the hippocampal surface could be examined in all subjects using the zones predefined on the surface of the provisory hippocampal template.

Left and right hippocampal volumes in each subject were calculated as the volumes enclosed by the hippocampal surfaces. An average hippocampal surface previously constructed from 86 healthy subjects was used as a reference surface [12], from which normal deformation of each subject's hippocampal surface was calculated at each surface point. For each subject, deformations were averaged within each surface zone to represent surface deformations for CA1, subiculum, and remainder subfields. Using the mean and standard deviation of the nondemented subjects' subfield deformation measures, we computed the $z$-scores of each subfield zone for each subject in the current study. Negative values of the surface measures represented inward deformation of the surface while positive values represented 
TABLE 1: Characteristics of the three groups.

\begin{tabular}{lcccc}
\hline & HS & AD & NC & Pvalues \\
\hline$N$ & 11 & 24 & 8 & \\
Age (s.d.) & $83.8(6.7)$ & $80.9(7.9)$ & $81.3(6.6)$ & .54 \\
Sex (F/M) & $3 / 8$ & $7 / 17$ & $6 / 2$ & .07 \\
Education (years) (s.d.) & $13(2.5)$ & $14.4(3.5)$ & $14.9(4.5)$ & .45 \\
Interval last MRI-death (y) (s.d.) & $1.8(0.95)$ & $3.2(1.9)$ & $3.7(2.6)$ & .06 \\
MMSE closest to death (s.d.) & $17.1(8.2)$ & $12.6(8.5)$ & $29.1(1.4)$ & $<.001$ \\
Duration of illness (years) (s.d.) & $7.1(4.5)$ & $8.6(3.5)$ & - & .14 \\
Braak \& Braak score (0-6) & $1.7(1.2)$ & $5.6(0.5)$ & $1.1(1)$ & $<.001$ \\
CERAD score (0-3) & $0.7(0.6)$ & $2.5(0.7)$ & $0.5(0.7)$ & $<.001$ \\
\hline
\end{tabular}
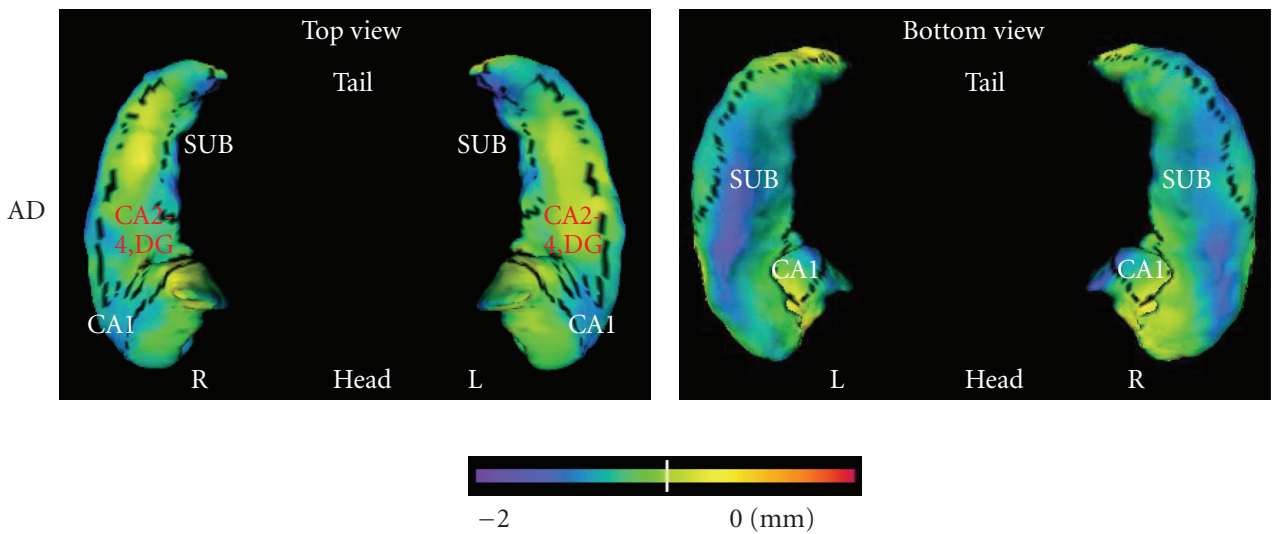

(a)

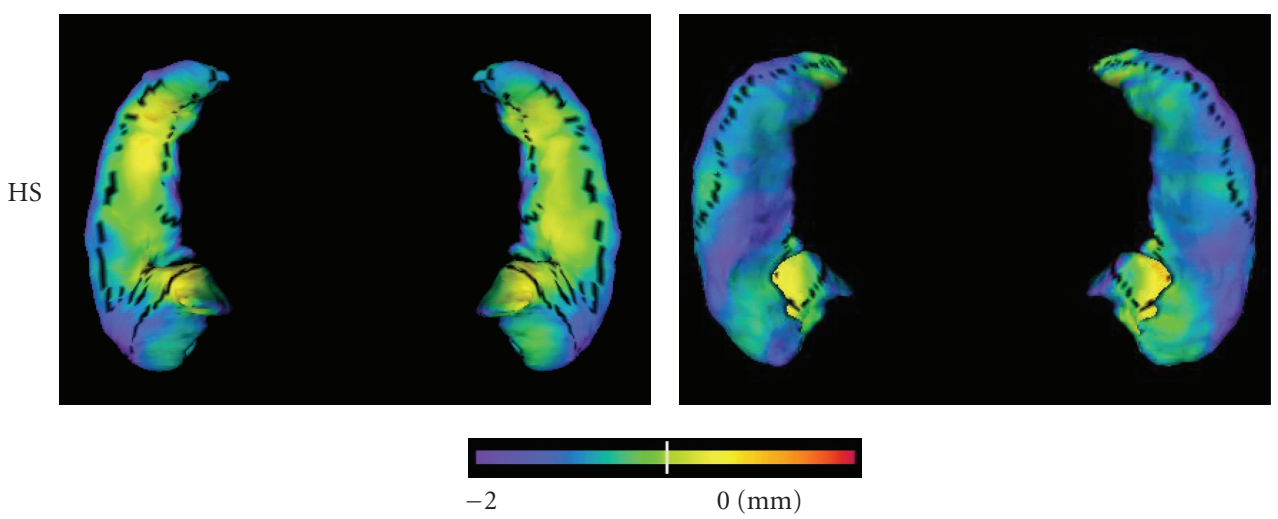

(b)

FIgure 1: Hippocampal surface deformities in AD (a) and HS (b) compared to NC. Right and left hippocampi are viewed from the top (dorsal surface, left panel) or the bottom (ventral surface, right panel). The flame scale (b) represents the difference between the mean surface of the disease hippocampus and the mean surface of NC hippocampus. Inward deformations are represented by cooler colors (blue to purple), while outward deformations are represented by warmer colors (orange to red). Green to yellow represent near zero deformations. Maximum deformation is $2 \mathrm{~mm}$ in either direction. The lateral surface (labeled CA1) is proximal to CA1, where the greatest deformations are found. Labels: SUB = subiculum, CA2-4, DG = combined fields of CA2, CA3, CA4, and the dentate granule cell layer.

outward deformation of the surface. Table 4 summarizes the outcomes of these three groups.

2.3. Statistical Analysis. One-way analysis of variance (ANOVA) was used to compare all of the demographic characteristics in Table 1 except for sex. Fisher's Exact Test was used to analyze the categorical variable sex. Two-way ANOVA using diagnostic group and sex as factors were used to compare hippocampal shape deformations and hippocampal volumes (Tables 4 and 5). Tukey-Kramer follow-up multiple comparison test was used if overall group differences were found. Planned comparisons of HS versus AD were carried out using least square means for hippocampal deformations 
Table 2: Dementia severity, clinical diagnoses, and pathological diagnoses for HS cases.

\begin{tabular}{|c|c|c|c|c|}
\hline Case & $\begin{array}{c}\text { Duration of } \\
\text { illness (years) }\end{array}$ & $\begin{array}{l}\text { Final } \\
\text { MMSE }\end{array}$ & $\begin{array}{c}\text { Final clinical } \\
\text { diagnosis }\end{array}$ & $\begin{array}{c}\text { Pathological } \\
\text { diagnosis }\end{array}$ \\
\hline 1 & 11 & 15 & $\mathrm{AD}$ & HS \\
\hline 2 & 4 & 18 & $\mathrm{AD}$ & HS \\
\hline 3 & 5 & 3 & $\mathrm{AD}$ & HS + IVD \\
\hline 4 & 1 & 27 & CVD & HS + IVD \\
\hline 5 & 5 & 22 & DLB & $\begin{array}{c}\mathrm{HS}+\mathrm{DLB}+ \\
\text { IVD }\end{array}$ \\
\hline 6 & 17 & 6 & FTD & $\mathrm{HS}+\mathrm{FTD}$ \\
\hline 7 & 4 & 28 & IVD & HS \\
\hline 8 & 8 & 25 & $\begin{array}{c}\text { possible } \\
\text { AD/possible } \\
\text { IVD }\end{array}$ & HS \\
\hline 9 & 5 & 14 & $\begin{array}{c}\text { possible } \\
\text { AD/possible } \\
\text { IVD }\end{array}$ & $\mathrm{HS}+\mathrm{IVD}$ \\
\hline 10 & 7 & 12 & $\begin{array}{c}\text { possible } \\
\text { AD/possible } \\
\text { IVD }\end{array}$ & HS +IVD \\
\hline 11 & 11 & 18 & $\begin{array}{c}\text { possible } \\
\text { AD/possible } \\
\text { IVD }\end{array}$ & HS + IVD \\
\hline
\end{tabular}

and hippocampal volumes. Analyses were two-tailed with the significance level set at $P<.05$ and were carried out with the interactive software SAS 9.1 (SAS Institute, Cary, NC).

\section{Results and Discussion}

3.1. Case Selection. Because HS in the context of AD presents a confound due to hippocampal neuron loss as a result of both HS and tangle formation, the HS cases included in this analysis were selected to have HS without concomitant AD. Similarly, AD cases with accompanying HS were excluded.

3.2. Demographic Characteristics (Table 1). The 3 comparison groups were comparable in age (81 to 84 years) and education ( 13 to 15 years). The NC group was predominantly female, which limited our ability to match with AD and HS cases on sex. The interval between last MRI and death was shorter in the HS group. Although the mean minimental status exam (MMSE) score was lower in AD (12.6) compared to HS (17.1) this difference was not statistically significant.

Table 2 details the duration of illness, the final MMSE score, the clinical diagnosis, and the pathological diagnosis for the $11 \mathrm{HS}$ cases. None of these cases was diagnosed clinically with HS. Table 3 lists the dementia severity, clinical diagnosis, and pathological diagnosis for the $\mathrm{AD}$ cases, most of which were clinically diagnosed with AD.

3.3. Hippocampal Surface Deformations. Figure 1 illustrates the hippocampal surface deformations for AD hippocampus and HS hippocampus compared to NC hippocampus. To aid
TABle 3: Dementia severity, clinical diagnoses, and pathological diagnoses for $\mathrm{AD}$ cases.

\begin{tabular}{|c|c|c|c|c|}
\hline Case & $\begin{array}{c}\text { Duration of } \\
\text { illness (years) }\end{array}$ & $\begin{array}{c}\text { Final } \\
\text { MMSE }\end{array}$ & $\begin{array}{c}\text { Final clinical } \\
\text { diagnosis }\end{array}$ & $\begin{array}{c}\text { Pathological } \\
\text { diagnosis }\end{array}$ \\
\hline 1 & 10 & 20 & $\mathrm{AD}$ & $\mathrm{AD}$ \\
\hline 2 & 4 & 6 & $\mathrm{AD}$ & $\mathrm{AD}$ \\
\hline 3 & 5 & 10 & $\mathrm{AD}$ & $\mathrm{AD}$ \\
\hline 4 & 10 & 17 & $\mathrm{AD}$ & $\mathrm{AD}$ \\
\hline 5 & 9 & 21 & $\mathrm{AD}$ & $\mathrm{AD}$ \\
\hline 6 & 10 & 0 & $\mathrm{AD}$ & $\mathrm{AD}$ \\
\hline 7 & 10 & 4 & $\mathrm{AD}$ & $\mathrm{AD}$ \\
\hline 8 & 7 & 15 & $\mathrm{AD}$ & $\mathrm{AD}$ \\
\hline 9 & 10 & 3 & $\mathrm{AD}$ & $\mathrm{AD}$ \\
\hline 10 & 6 & 8 & $\mathrm{AD}$ & $\mathrm{AD}$ \\
\hline 11 & 5 & 20 & $\mathrm{AD}$ & $\mathrm{AD}$ \\
\hline 12 & 14 & 3 & $\mathrm{AD}$ & $\mathrm{AD}$ \\
\hline 13 & 9 & 2 & $\mathrm{AD}$ & $\mathrm{AD}$ \\
\hline 14 & 8 & 18 & CVD & $\mathrm{AD}$ \\
\hline 15 & 10 & 0 & FTD & $\mathrm{AD}$ \\
\hline 16 & 0 & 25 & MCI & $\mathrm{AD}$ \\
\hline 17 & 6 & 20 & $\mathrm{AD} / \mathrm{IVD}$ & $\mathrm{AD}$ \\
\hline 18 & 9 & 0 & AD/IVD & $\mathrm{AD}$ \\
\hline 19 & 7 & 20 & AD/IVD & $\mathrm{AD}$ \\
\hline 20 & 15 & 18 & AD/IVD & $\mathrm{AD}$ \\
\hline 21 & 5 & 12 & AD/IVD & $\mathrm{AD}$ \\
\hline 22 & 14 & 22 & AD/IVD & $\mathrm{AD}$ \\
\hline 23 & 13 & 14 & $\mathrm{AD}$ & $\mathrm{AD}$ \\
\hline 24 & 10 & 24 & MCI & $\mathrm{AD}$ \\
\hline
\end{tabular}

the visual identification of the three zones of the hippocampal surface (i.e., CA1, subiculum, and the combined fields of CA2, CA3, CA4, and the dentate granule cell layer) the boundaries that demarcate them are shown black. For the comparison between $\mathrm{AD}$ and the NC (Figure 1(a)), areas of hippocampus showing the greatest group differences (as marked by the blue colors) are concentrated in the CA1 and subiculum surface zones. These patterns of deformation resemble our previous findings in nonoverlapping subjects [12]. The comparison between HS and NC hippocampi (Figure 1(b)) shows a deformation pattern with similar extent but increased magnitude (in the negative or inward direction) than that between $\mathrm{AD}$ and NC. This observation was further supported by statistical comparison of surface deformation $z$-scores (Table 4).

Quantitative hippocampal surface deformations expressed as z-scores are presented in Table 4. In comparing $\mathrm{AD}$ to $\mathrm{NC}$, there were significant inward deformations of right CA1, left subiculum, right subiculum, and left and right subiculum combined (total) $(P=.001)$. As shown in Figure 1, differences were found in the CA1 and subiculum surface zone deformations but not in the $\mathrm{CA} 2+\mathrm{CA} 3+\mathrm{CA} 4+\mathrm{DG}$ zone. There was no interaction with sex for any of the comparisons. 
TABLE 4: Hippocampal shape deformations expressed as $z$-scores.

\begin{tabular}{|c|c|c|c|c|}
\hline & $\begin{array}{c}\text { NC } \\
(n=8)\end{array}$ & $\begin{array}{c}\mathrm{AD} \\
(n=24)\end{array}$ & $\begin{array}{c}\text { HS } \\
(n=11)\end{array}$ & $F(2,40), P$ \\
\hline \multicolumn{5}{|c|}{ CA1 Deformation } \\
\hline Left & $-0.31(0.50)$ & $-0.90(0.59)$ & $-1.54(1.08)^{1}$ & $F=6.69, P=.003$ \\
\hline Right & $-0.35(0.44)$ & $-1.07(0.68)^{1}$ & $-1.74(0.85)^{1,2}$ & $F=9.37, P=.005$ \\
\hline Total & $-0.33(0.42)$ & $-0.99(0.60)$ & $-1.64(0.91)^{1,2}$ & $F=9.02, P=.006$ \\
\hline \multicolumn{5}{|c|}{ Subicular Deformation } \\
\hline Left & $0.06(0.19)$ & $-0.39(0.21)^{1}$ & $-0.73(0.41)^{1,2}$ & $F=19.14, P<.001$ \\
\hline Right & $0.09(0.21)$ & $-0.34(0.22)^{1}$ & $-0.67(0.28)^{1,2}$ & $F=23.93, P<.001$ \\
\hline Total & $0.08(0.19)$ & $-0.36(0.20)^{1}$ & $-0.70(0.31)^{1,2}$ & $F=25.71, P<.001$ \\
\hline \multicolumn{5}{|c|}{ Combined deformation } \\
\hline Left & $0.24(0.31)$ & $-0.04(0.33)$ & $0.007(0.39)$ & $F=2.01, P=.14$ \\
\hline Right & $0.08(0.22)$ & $-0.17(0.29)$ & $-0.15(0.33)$ & $F=2.30, P=.11$ \\
\hline Total & $0.16(0.25)$ & $-0.11(0.27)$ & $-0.07(0.30)$ & $F=2.88, P=.07$ \\
\hline
\end{tabular}

${ }^{1}$ Different from NC, Tukey-Kramer $(P<.05)$.

${ }^{2}$ Different from AD, Tukey-Kramer $(P<.05)$.

TABLE 5: Left, right, and total hippocampal volumes in NC, AD, and HS.

\begin{tabular}{lcccc}
\hline & & \multicolumn{2}{c}{ Hippocampal volume } & HS \\
\hline Left & NC & AD & $1294.8(535.6)^{1,2}$ & $F(2,40), P$ \\
Right & $2170.2(333.8)$ & $1638.1(305.7)^{1}$ & $F=12.27, P<.0001$ \\
Total & $2235.5(259.7)$ & $1622.8(341.5)^{1}$ & $1251.9(352.0)^{1,2}$ & $F=20.49, P<.0001$ \\
\hline
\end{tabular}

Data are mean (s.d.) in: $\mu \mathrm{m}^{3}$.

${ }^{1}$ Different from NC, Tukey-Kramer $(P<.05)$.

${ }^{2}$ Different from AD, Tukey-Kramer $(P<.05)$.

In comparing $\mathrm{HS}$ with $\mathrm{NC}$, there were significant inward deformations of left CA1, right CA1, left subiculum, right subiculum, and left and right subiculum combined (total) $(P=.001)$.

In comparing $\mathrm{HS}$ with $\mathrm{AD}$, significant inward deformations were found for right CA1, and left and right CA1 combined (total), left subiculum, right subiculum, and left and right subiculum combined (total) $(P=.001)$.

The CA1 surface zone deformations of the NC subjects in this study showed an appreciable amount of inward deformation (left -0.31 , right -0.35 ) while the healthy reference group from Wang et al. [12] had an average of 0 (used as reference). This difference could be due to the fact that 20 healthy subjects from a study of schizophrenia were included in the reference group of the previous study. The subjects included in the present study were about 10 years older, and aging may be related to hippocampal volume loss and shape deformities. A similar phenomenon was observed in another study of more elderly subjects with dementia of the Alzheimer type [14].

3.4. Hippocampal Volume (Table 5). There were no statistically significant differences in right versus left hippocampal volume for any group. However, there were statistically significant differences between $\mathrm{NC}$ compared to $\mathrm{AD}, \mathrm{NC}$ compared to HS, and AD compared to HS $(P<.001)$. On average, hippocampal volumes for $\mathrm{AD}$ were $25.9 \%$ less than
NC. HS hippocampal volumes were $21.9 \%$ less than AD and $42.2 \%$ less than NC. There was no interaction with sex for any of the comparisons.

\section{Conclusions}

In this paper we demonstrate that hippocampal atrophy and the corresponding changes in hippocampal shape distinguish HS from $\mathrm{AD}$ on premortem MRI. More atrophy and greater deformation were observed in those portions of the hippocampal surface in proximity to the CA1 and subiculum subfields in HS compared to AD. These results support the possibility that greater severity of hippocampal atrophy and deformation may be useful for the clinical identification of HS. Additional studies will be needed, however, to determine whether severity of atrophy and deformation can be used to distinguish HS from AD on an individual case basis. Better criteria for clinical diagnosis are needed before advances can be made in understanding and preventing HS in late life.

We surmise that the significant inward deformation of CA1 found in this study is probably due to neuronal loss as a result of neurofibrillary degeneration in $\mathrm{AD}$ and to unknown pathogenesis in HS. Neuron loss in AD is well documented, and although tangles represent dying or dead neurons, the tangle itself is space-filling, that is, it takes up a volume within the hippocampal subfield, presumably smaller than the healthy neuron. Although significant neuron loss occurs 
in $\mathrm{AD}$, there are large numbers of neurons remaining [10]. In contrast, $90 \%$ of hippocampal neurons may be lost in HS. Microscopically, it appears that the neuron loss in HS involves primarily CA1 and sometimes subiculum. However, if this condition were restricted to these regions, the volume losses would not be as great as those seen here. In other words, the mechanism leading to the overall volume loss seen in HS affects the entire hippocampus.

A clinical diagnosis of HS is rarely if, ever, made. Indeed, none of the HS cases in this study received a diagnosis of HS. Three cases were clinically diagnosed with possible AD, 2 with possible IVD, 1 with probable DLB, 1 FTD, and 4 were diagnosed with mixed possible AD/possible IVD. A specific biomarker for the clinical detection and diagnosis of HS is greatly needed.

Previous studies have demonstrated a lack of substantial loss of hippocampal volume in healthy elderly control subjects as compared with the younger control subjects [15]. In this study, we excluded cases of AD with HS. Had these cases had been included, the differences between the groups would have been less. Age, years of education, MMSE score, and duration of illness were not significantly different in the two disease groups. The NC group was predominantly female. It is recognized that brain and hippocampal volumes are generally smaller in females compared to males. The predominance of females in the $\mathrm{NC}$ group would have affected the AD to NC and HS to NC comparison equally, and might have led to an underestimation of volume differences. However, we did not find any effect of sex on any of these analyses. The shorter interval between MRI and death in the HS group may have contributed to the increased severity of atrophy in the HS group, assuming that atrophy is an equally progressive process in $\mathrm{AD}$ and $\mathrm{HS}$. While the group differences in mean severity of hippocampal atrophy and shape deformation are significant, additional study is needed to determine whether these differences will be meaningful for clinical diagnosis of individuals.

\section{Acknowledgments}

C. Zarow had full access to all of the data in the study and takes responsibility for the integrity of the data and the accuracy of the data analysis. This work was supported by 1P01 AG12435, 1P50 AG05142.

\section{References}

[1] J. B. Leverenz, C. M. Agustin, D. Tsuang et al., "Clinical and neuropathological characteristics of hippocampal sclerosis: a community-based study," Archives of Neurology, vol. 59, no. 7, pp. 1099-1106, 2002.

[2] C. Zarow, T. E. Sitzer, and H. C. Chui, "Understanding hippocampal sclerosis in the elderly: epidemiology, characterization, and diagnostic issues," Current Neurology and Neuroscience Reports, vol. 8, no. 5, pp. 363-370, 2008.

[3] W. J. Jagust, L. Zheng, D. J. Harvey et al., "Neuropathological basis of magnetic resonance images in aging and dementia," Annals of Neurology, vol. 63, no. 1, pp. 72-80, 2008.

[4] K. A. Josephs, J. L. Whitwell, C. R. Jack, J. E. Parisi, and D. W. Dickson, "Frontotemporal lobar degeneration without lobar atrophy," Archives of Neurology, vol. 63, no. 11, pp. 1632-1638, 2006.

[5] M. F. Mendez, T. Chow, J. Ringman, G. Twitchell, and C. H. Hinkin, "Pedophilia and temporal lobe disturbances," Journal of Neuropsychiatry and Clinical Neurosciences, vol. 12, no. 1, pp. 71-76, 2000.

[6] F. Barkhof, T. M. Polvikoski, E. C. W. van Straaten et al., "The significance of medial temporal lobe atrophy: a postmortem MRI study in the very old," Neurology, vol. 69, no. 15, pp. 1521-1527, 2007.

[7] J. G. Csernansky, L. Wang, S. C. Joshi, J. T. Ratnanather, and M. I. Miller, "Computational anatomy and neuropsychiatric disease: probabilistic assessment of variation and statistical inference of group difference, hemispheric asymmetry, and time-dependent change," NeuroImage, vol. 23, no. 1, pp. S56S68, 2004.

[8] L. Wang, J. S. Swank, I. E. Glick et al., "Changes in hippocampal volume and shape across time distinguish dementia of the Alzheimer type from healthy aging," NeuroImage, vol. 20, no. 2, pp. 667-682, 2003.

[9] J. G. Csernansky, L. Wang, J. Swank et al., "Preclinical detection of Alzheimer's disease: hippocampal shape and volume predict dementia onset in the elderly," NeuroImage, vol. 25, no. 3, pp. 783-792, 2005.

[10] C. Zarow, H. V. Vinters, W. G. Ellis et al., "Correlates of hippocampal neuron number in Alzheimer's disease and ischemic vascular dementia," Annals of Neurology, vol. 57, no. 6, pp. 896-903, 2005.

[11] Y. Y. Hsu, N. Schuff, A. T. Du et al., "Comparison of automated and manual MRI volumetry of hippocampus in normal aging and dementia," Journal of Magnetic Resonance Imaging, vol. 16, no. 3, pp. 305-310, 2002.

[12] L. Wang, J. P. Miller, M. H. Gado et al., "Abnormalities of hippocampal surface structure in very mild dementia of the Alzheimer type," NeuroImage, vol. 30, no. 1, pp. 52-60, 2006.

[13] M. Vaillant and J. Glaunès, "Surface matching via currents," Information Processing in Medical Imaging, vol. 19, pp. 381392, 2005.

[14] L. Wang, A. Khan, J. G. Csernansky et al., "Fully-automated, multi-stage hippocampus mapping in very mild Alzheimer disease," Hippocampus, vol. 19, no. 6, pp. 541-548, 2009.

[15] J. G. Csernansky, L. Wang, S. Joshi et al., "Early DAT is distinguished from aging by high-dimensional mapping of the hippocampus. Dementia of the Alzheimer type," Neurology, vol. 55, no. 11, pp. 1636-1643, 2000. 


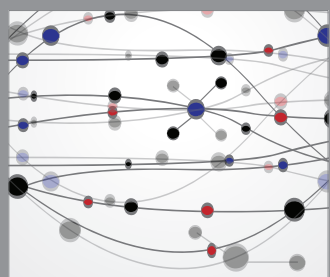

The Scientific World Journal
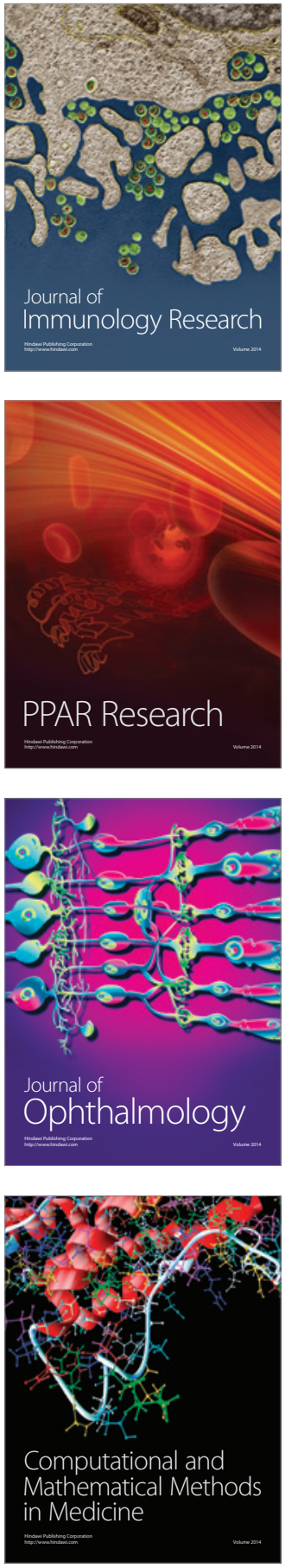

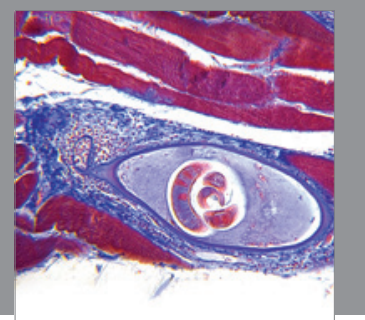

Gastroenterology

Research and Practice
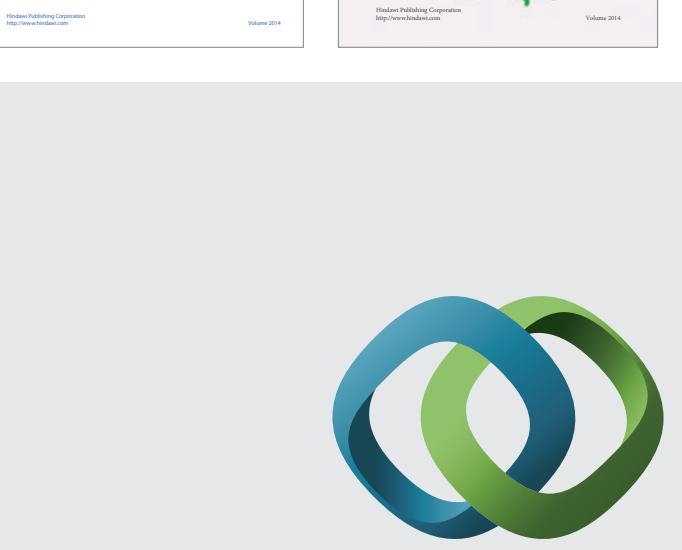

\section{Hindawi}

Submit your manuscripts at

http://www.hindawi.com
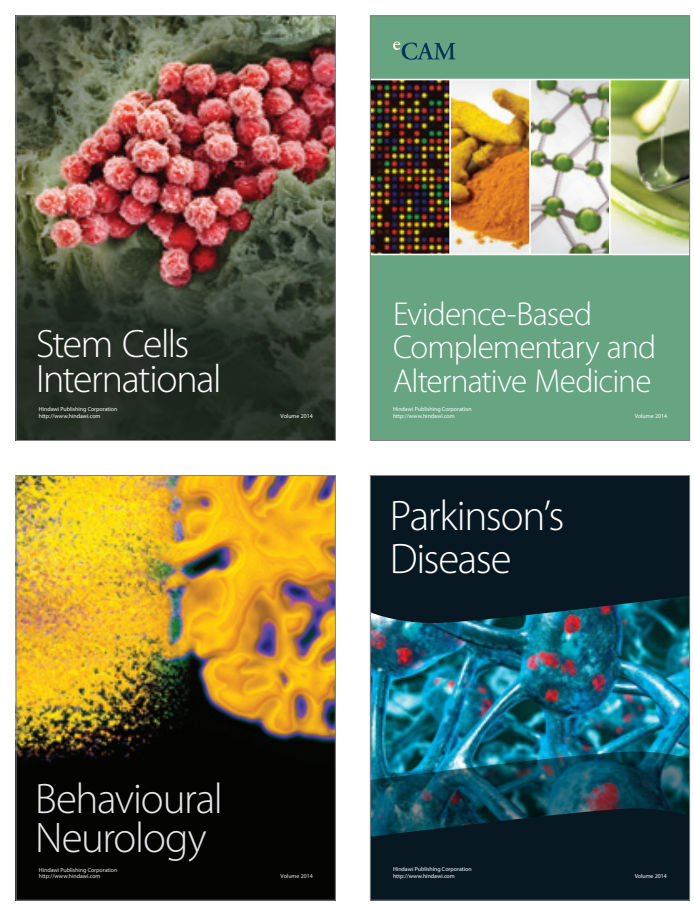

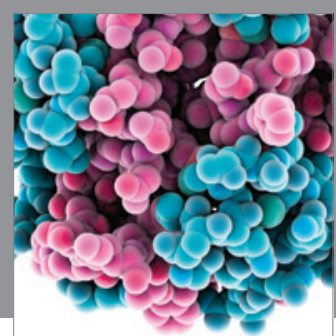

Journal of
Diabetes Research

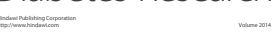

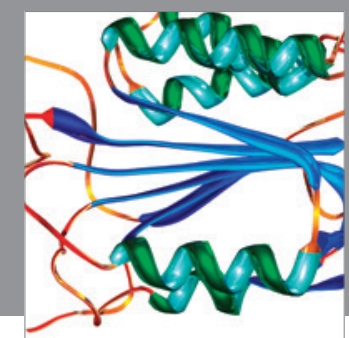

Disease Markers
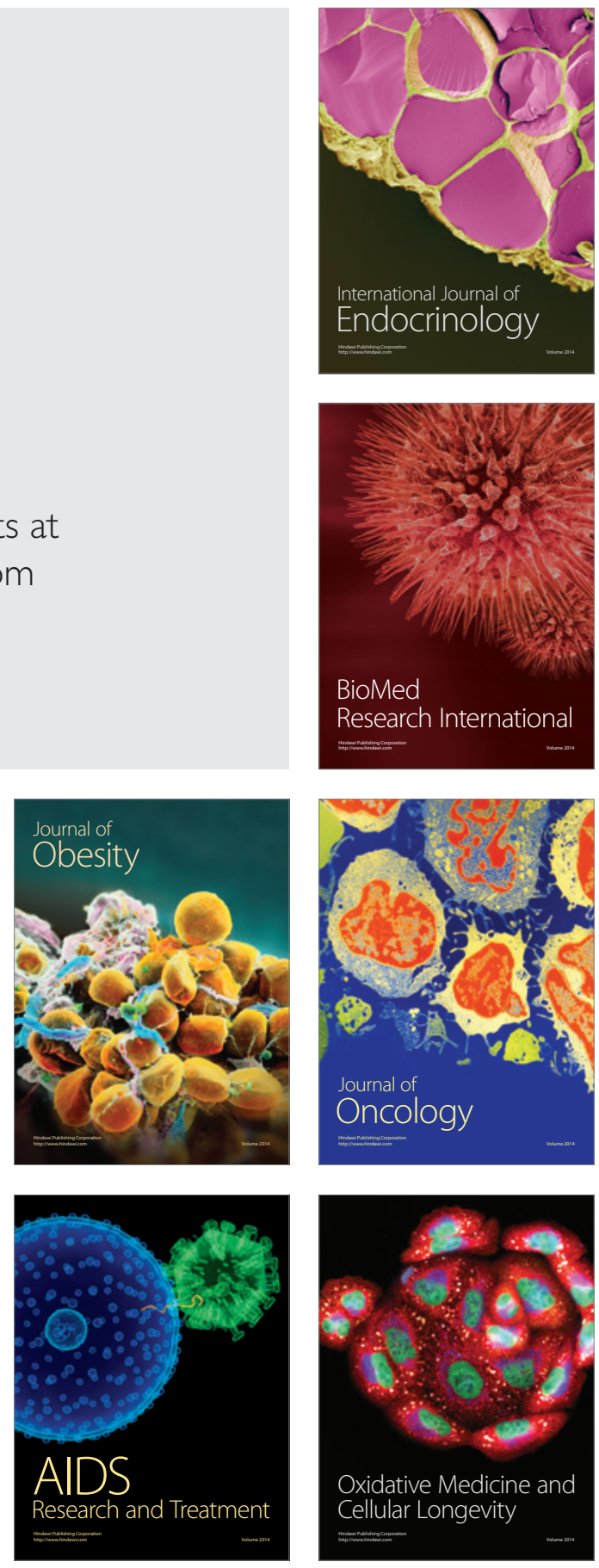